Ricardo Morrot Lima

\title{
Simulação Tridimensional em Tempo Real de Veículos Robóticos em Terrenos Acidentados
}

Dissertação apresentada ao Programa de PósGraduação em Engenharia Mecânica da PUC-Rio como requisito parcial para obtenção do título de Mestre em Engenharia Mecânica.

Orientador: Prof. Marco Antonio Meggiolaro 
Ricardo Morrot Lima

\title{
Simulação Tridimensional em Tempo Real de Veículos Robóticos em Terrenos Acidentados
}

\begin{abstract}
Dissertação apresentada como requisito parcial para obtenção do grau de Mestre pelo Programa de Pósgraduação em Engenharia Mecânica da PUC-Rio. Aprovada pela Comissão Examinadora abaixo assinada.
\end{abstract}

Prof. Marco Antonio Meggiolaro

Orientador

Departamento de Engenharia Mecânica - PUC-Rio

Prof. Hans Ingo Weber

Departamento de Engenharia Mecânica - PUC-Rio

Prof. Mauro Speranza Neto

Departamento de Engenharia Mecânica - PUC-Rio

Prof. José Eugenio Leal

Coordenador Setorial do Centro

Técnico Científico - PUC-Rio

Rio de Janeiro, 29 de setembro de 2010 
Todos os direitos reservados. É proibida a reprodução total ou parcial do trabalho sem autorização da universidade, do autor e do orientador.

\section{Ricardo Morrot Lima}

Graduou-se em Desenho Industrial - Projeto de Produto pela Pontifícia Universidade Católica do Rio de Janeiro em 1997. Pós-Graduação em Análise, Projeto e Gerência de Sistemas pela Pontifícia Universidade Católica do Rio de Janeiro em 2000.

Ficha Catalográfica

Lima, Ricardo Morrot
Simulação tridimensional em tempo real de veículos
robóticos em terrenos acidentados / Ricardo Morrot Lima;
orientador: Marco Antonio Meggiolaro. - 2010.
170 f. : il. (color.) ; $30 \mathrm{~cm}$
Dissertação (mestrado) - Pontifícia Universidade
Católica do Rio de Janeiro, Departamento de Engenharia
Mecânica, 2010.
Inclui bibliografia
1. Engenharia mecânica - Teses. 2. Simulação. 3.
Tridimensional. 4. 3D. 5. Tempo real. 6. Veículos
robóticos. 7. Terrenos acidentados. 8. Controle de
estabilidade. 9. Dinâmica veicular. 10. LuGre. I.
Meggiolaro, Marco Antonio. II. Pontifícia Universidade
Católica do Rio de Janeiro. Departamento de Engenharia
Mecânica. III. Título.

CDD: 621 
Não haveria um segundo da minha vida que eu não dedicasse a Deus, e este trabalho só foi possível porque Ele o permitiu, à minha querida mãe, Luiza, que me passou toda essa perseverança de vida, aos meus irmãos, Alexandre e Mônica, de quem tenho bastante orgulho, e à minha querida sobrinha, Katheryn, que estará eternamente no meu coração. 


\section{Agradecimentos}

Tenho muito a agradecer.

Agradeço primeiramente ao professor Mauro Speranza Neto pela atenção dada, por ter ouvido a minha intenção e me indicado ao professor Marco Antonio Meggiolaro, meu orientador.

Sinto-me feliz por poder retribuir formalmente ao meu orientador Marco Antonio Meggiolaro pelo desafio a que foi submetido, pela paciência, pelas vastas horas que lhe roubei e, acima de tudo, pela confiança depositada em mim, mantida mesmo nas dificuldades.

Gostaria de agradecer especialmente ao professor Hans Ingo Weber por ter acreditado em mim e por ter me impulsionado para o universo maravilhoso da dinâmica.

Não há como deixar de agradecer a uma figura essencial, sem a qual a jornada seria literalmente um "terreno acidentado": obrigado ao amigo Pedro Blois pela ajuda técnica, científica, pela força e conexão estabelecida.

Aos amigos César Raúl Mamani Choquehuanca, Danny Hernán Zambrano, Cristian Mejia Sanchez e a todos os que colaboraram direta ou indiretamente para a concretização deste sonho.

A toda galera do Tecgraf/MVGEO representada pelo professor Luís Fernando Martha e, em especial, a ele.

Ao CNPq e à CAPES pelo apoio financeiro durante o curso de mestrado.

Meus sinceros agradecimentos. 


\section{Resumo}

Lima, Ricardo Morrot; Meggiolaro, Marco Antonio. Simulação Tridimensional em Tempo Real de Veículos Robóticos em Terrenos Acidentados. Rio de Janeiro, 2010. 170p. Dissertação de Mestrado Departamento de Engenharia Mecânica, Pontifícia Universidade Católica do Rio de Janeiro.

Esta dissertação aborda conceitos interdisciplinares de Engenharia Mecânica e Engenharia de Software, com foco principal no estudo de sistemas mecânicos. Atualmente, operações de monitoração por meio de veículos autônomos se tornam cada vez mais comuns, enquanto os ambientes a que esses veículos robóticos são submetidos passam a ser cada vez mais hostis, principalmente em relação aos obstáculos e características dos terrenos. O presente trabalho introduz o desenvolvimento de um simulador dinâmico em 3D em tempo real para veículos robóticos em terrenos acidentados. Um algoritmo de interseção é desenvolvido entre um terreno 3D genérico e cada roda de um veículo. Um modelo de força de contato pneu-terreno é implementado, levando em consideração as combinações das derivas longitudinal e lateral. O modelo também inclui os efeitos de corrente contínua de motores, levando-se em consideração a interação entre a parte mecânica e a elétrica, inclusive uma aproximação contínua do modelo de atrito de LuGre, considerando as limitações de potência das baterias do sistema. O simulador também inclui equações para um controle de estabilidade 2D, levando em consideração apenas a estabilização do ângulo de arfagem (pitch) do veículo. Este trabalho propõe, além disso, um controle de estabilidade 3D utilizando um indicador de estabilidade que pode ser calculado em tempo real, baseado em uma estimativa de distribuição de forças de contato entre roda e terreno. O simulador é validado mediante comparações com soluções analíticas do comportamento longitudinal do veículo robótico.

\section{Palavras-chave}

Simulação; Tridimensional; 3D; Tempo Real; Veículos Robóticos; Terrenos Acidentados; Controle de Estabilidade; Dinâmica Veicular; LuGre. 


\section{Abstract}

Lima, Ricardo Morrot; Meggiolaro, Marco Antonio. Three-dimensional Simulation in Real Time of Mobile Robotics on Rough Terrain. Rio de Janeiro, 2010. 170pp. MSc. Dissertation - Departamento de Engenharia Mecânica, Pontifícia Universidade Católica do Rio de Janeiro.

This dissertation approaches interdisciplinary concepts of Mechanical Engineering and Software Engineering, with a main focus on the study of mechanical systems. Nowadays, the task of monitoring with autonomous vehicles has become more and more common, while the environment to which those robot vehicles are exposed becomes more and more hostile, mainly in relation to the obstacles and characteristics of the terrain. The present work introduces the development of a 3D real-time dynamic simulator of robot vehicles on rough terrain. An intersection algorithm is developed between a 3D generic terrain and each wheel of a vehicle. A tire-soil contact force model is implemented, taking into consideration the combined longitudinal and lateral drifts. The model includes the effects of direct current motors, taking into consideration the interaction between mechanical and electric parts, including a continuous approximation of LuGre's friction model, considering the power limitations of the system batteries. The simulator also includes an equation for a 2D stability control, taking into consideration only the stabilization of the pitch angle of the vehicle. This work also proposes a 3D stability control using an indicator of stability that can be calculated in real time, based on an estimated distribution of wheel-terrain contact forces. The simulator is validated through comparisons with analytic solutions of the longitudinal behavior of the robot vehicle.

\section{Keywords}

Simulation; Three-dimensional; 3D; Real Time; Mobile Robots; Rover; Rough Terrain; Traction Control; Vehicle Dynamics; LuGre. 


\section{Sumário}

1 Introdução 20

1.1. Definição de Rover 24

1.2. Definição de Robô Móvel (Mobile Robot) 25

1.3. Objetivo 26

1.4. Estrutura da Dissertação 26

2 Revisão Bibliográfica 28

2.1. Controle de Tração em Terrenos Acidentados 28

2.2. Simuladores Avaliados 29

2.3. Arquitetura dos Simuladores Avaliados 38

2.4. Biblioteca Gráfica dos Simuladores Avaliados 41

2.5. Biblioteca Utilizada na Dinâmica dos Simuladores Avaliados 42

3 Simulador Desenvolvido $\quad 45$

3.1. Descrição Geral 47

3.2. Modelagem do Terreno 49

3.3. Modelagem do Veículo Robótico 52

3.3.1. Parâmetros Geométricos do Veículo Robótico 52

3.3.2. Forças Externas sobre o Veículo Robótico 54

3.3.3. Forças Internas entre a Roda e o Chassi 55

3.3.4. Algoritmo de Busca do Ponto de Contato entre Roda e Terreno 56

3.4. Modelagem Dinâmica do Veículo Robótico 61

3.5. Modelo da Força de Contato Pneu-Terreno 66

3.5.1. Derivas Longitudinal e Lateral 66

3.5.2. Cálculo das Forças Longitudinal e Lateral 67

3.5.3. Combinação das Derivas $\quad 71$

3.6. Atuador 71

$\begin{array}{ll}\text { 3.7. Método de Integração } & 73\end{array}$

$\begin{array}{ll}4 \text { Controle } & 74\end{array}$ 
5 Validação do Software $\quad 81$

5.1. Validação da Simulação da Arrancada 81

5.2. Validação da Simulação do Salto 85

6 Resultados $\quad 88$

6.1. Controle Proporcional Simples em Terreno Senoidal 92

6.2. Controle CDTA em Terreno Senoidal 95

6.3. Controle Proporcional Simples em Terreno Plano $\begin{array}{ll}\text { com uma Rampa } & 97\end{array}$

6.4. Controle CDTA em Terreno Plano com uma Rampa 100

7 Conclusões 104

8 Referências bibliográficas 106

Anexo A: $\quad$ Organização dos Arquivos do Aplicativo VirtualBotz 3D 113

Anexo B: Diagrama de Classes (UML) do Simulador 115

Anexo C: Comandos do Joystick (Analógico) 121

Anexo D: Teclas de Funcionalidades do Simulador 123

D.1. Terminar execução do simulador 123

D.2. Alternar visão da simulação pelas câmeras

interna e externas do veículo robótico 123

$\begin{array}{ll}\text { D.3. Visualizar legendas } & 126\end{array}$

D.4. Encerrar (parar) simulação corrente 126

D.5. Iniciar nova simulação 126

$\begin{array}{ll}\text { D.6. Salvar simulação corrente em vídeo } & 127\end{array}$

D.7. Visualizar trajetória do centro de massa do veículo robótico $\begin{array}{ll}\text { após a simulação } & 129\end{array}$

D.8. Posicionar o veículo robótico pelo centro de massa ao longo da trajetória após a simulação 
Anexo E: Scripts de Configuração do Simulador

Anexo F: Opções de Terrenos 145

Anexo G: Configurando Opção de Terreno no Script da Simulação 151

Anexo H: Renderização do Terreno 153

Anexo I: Configurando o Veículo Robótico no Script da Simulação 155

Anexo J: Configurando os Motores das Rodas

do Veículo Robótico no Script da Simulação

Anexo K: Habilitando no Script da Simulação a Geração de Dados da Simulação Corrente do Veículo Robótico em Arquivos 


\section{Lista de Figuras}

Figura 1 - Protótipo desenvolvido pelo Grupo de Robótica

CENPES/Petrobras a ser utilizado na região amazônica

Figura 2 - Gasoduto Coari, Manaus (Barral, 2007, p. 20) [4] 24

Figura 3 - CLARAty navegando o Rocky 8 ROAMS Simulator [37] 30

Figura 4 - ROAMS, ou Rover Modeling and Simulation, produzido pelo

Jet Propulsion Laboratory da NASA, em uma missão de superfície [25]

Figura 5 - Universal Mechanisms simulando um veículo robótico

de seis rodas para transporte [12]

Figura 6 - Universal Mechanisms simulando a dinâmica de um utilitário

sobre uma lombada [12]

Figura 7 - Gazebo simulando multirrobôs em ambiente controlado,

modificado de [15]

Figura 8 - Um exemplo da configuração de rover no RCAST [16] 34

Figura 9 - CRAB Rover durante uma simulação [38] 35

Figura 10 - Aplicação robótica utilizando o simulador RDS [33] 36

Figura 11 - Ambiente visual do RDS para criação de

aplicações robóticas [33]

Figura 12 - Veículo passando sobre uma rampa unilateral,

SIMPACK Automotivo

Figura 13 - Arquitetura em camadas do CLARAty, modificado de [10]

Figura 14 - Exemplo, modificado de [27], ilustrando a hierarquia

de objetos e o conceito de herança de classes

Figura 15 - Diagrama da arquitetura do aplicativo Gazebo,

modificado de [60]

Figura 16 - Veículo para Inspeção Visual Interna de dutos (VIVI)

Figura 17 - Modelo de grade regular retangular utilizado pelo

VirtualBotz 3D

Figura 18 - Extremos do domínio do terreno no VirtualBotz 3D 50

Figura 19 - Ilustração das variáveis de interpolação do terreno 51

Figura 20 - Esquema com as dimensões do chassi 52 
Figura 21 - Centro de massa teórico da roda, sem qualquer

deformação na suspensão

Figura 22 - Dimensões da roda $\quad 54$

Figura 23 - Esquema de forças externas atuando sobre o veículo robótico $\quad 55$

Figura 24 - Esquema de forças interna entre a roda e o chassi 55

Figura 25 - Deslocamento imposto sobre a suspensão 56

Figura 26 - Ilustração da discretização da circunferência inferior da roda 57

Figura 27 - Ilustração do esquema do cálculo de $\boldsymbol{X}_{\boldsymbol{c}}$ ’ 58

Figura 28 - Ilustração da convergência do ponto de contato da roda com o terreno $\quad 59$

Figura 29 - Esquema do ponto de contato da roda com o terreno 61

Figura 30 - Esforços agindo entre a roda e o terreno 63

Figura 31 - Curva produzida pela versão original da

“Fórmula Mágica” em [53] 68

Figura 32 - Região admissível para as forças de tração nas rodas 1 e 2 [56] 75

Figura 33 - Combinações da região $D$ e $\Gamma[56] \quad 76$

Figura 34 - Pontos de interseção entre $\Gamma$ e o menor diamante das linhas

de mesma potência para os quatro casos básicos $\quad 77$

Figura 35 - Sistema de cálculo dos eixos de estabilidade 79

Figura 36 - Gráfico 2D do momento de estabilidade do veículo robótico

na tela de visualização do aplicativo VirtualBotz 3D

Figura 37 - Definição das grandezas utilizadas no cálculo da solução

analítica para o teste de arrancada do veículo robótico

Figura 38 - Definição das grandezas utilizadas no cálculo da solução

analítica para o teste de salto do veículo robótico $\quad 85$

Figura 39 - Perfil do terreno senoidal desenhado no MATLAB 89

Figura 40 - Início da simulação: cenário da primeira simulação,

com o veículo robótico VIVI, no terreno senoidal

Figura 41 - Veículo robótico VIVI no terreno senoidal superando

$\begin{array}{ll}\text { o primeiro aclive } & 90\end{array}$

Figura 42 - Perfil da rampa do terreno desenhado no MATLAB 91

Figura 43 - Cenário da segunda simulação com o veículo robótico

VIVI, no terreno plano com uma rampa ao final 
Figura 44 - Veículo robótico VIVI no início da rampa

na segunda simulação, no terreno plano com uma rampa

Figura 45 - Velocidade do centro de massa do veículo

com o controlador proporcional simples com compensação de gravidade

Figura 46 - Força normal com o controlador proporcional simples

com compensação de gravidade

Figura 47 - Gráfico da deriva longitudinal da roda traseira

com o controlador proporcional simples com compensação de gravidade

Figura 48 - Potência dos motores com o controlador proporcional

simples com compensação de gravidade

Figura 49 - Gráfico da velocidade do centro de massa do veículo

com o controle CDTA

Figura 50 - Força normal com o controle CDTA

Figura 51 - Gráfico da deriva longitudinal da roda traseira

com o controle CDTA

Figura 52 - Potência dos motores com o controle CDTA

Figura 53 - Gráfico da velocidade do centro de massa do veículo

com o controle proporcional simples em terreno plano com uma rampa

Figura 54 - Força normal com o controlador proporcional simples

em terreno plano com uma rampa

Figura 55 - Gráfico da deriva longitudinal da roda traseira

com o controle proporcional simples em terreno plano com uma rampa

Figura 56 - Potência dos motores com o controlador proporcional

simples em terreno plano com uma rampa

Figura 57 - Velocidade do centro de massa do veículo

com o controle CDTA em terreno plano com uma rampa

Figura 58 - Força normal com o controle CDTA em terreno plano

com uma rampa

Figura 59 - Deriva longitudinal da roda traseira com o controle CDTA

em terreno plano com uma rampa

Figura 60 - Potência dos motores com o controle CDTA

em terreno plano com uma rampa

Figura 61 - Diagrama de classes (UML) do terreno 
Figura 62 - Diagrama de classes (UML) do veículo robótico

Figura 63 - Diagrama de classes (UML) para matrizes $3 \times 3$ e vetores $3 \times 1 / 1 \times 3 \quad 120$

Figura 64 - Comandos dos manetes do joystick

Figura 65 - Comandos dos botões do joystick

Figura 66 - Teclas de funcionalidades do simulador

(teclado padrão internacional)

Figura 67 - Veículo robótico observado pela câmera virtual 1,

permitindo a movimentação do mundo virtual de forma independente

do veículo robótico

Figura 68 - Visão pela câmera virtual interna do veículo robótico

durante várias simulações com diferentes tipos de terrenos

Figura 69 - Imagem obtida pela câmera virtual externa

que acompanha o veículo robótico

Figura 70 - Janela de seleção do CoDec para geração do

vídeo AVI da simulação

Figura 71 - Imagem de um vídeo AVI gerado com a visão

do observador, externa ao veículo

Figura 72 - Imagem de um vídeo AVI gerado com a

câmera virtual interna do veículo robótico

Figura 73 - Imagem de um vídeo AVI gerado com a visão

da terceira câmera virtual do aplicativo VirtualBotz 3D, em uma

simulação com o tipo de piso similar ao dos pilotis da PUC-Rio

Figura 74 - Veículo robótico com os vetores $\mathbf{Z}$ do centro de massa

após o término da simulação

Figura 75 - Veículo robótico com os vetores $\boldsymbol{X}, \boldsymbol{Y}$ e $\boldsymbol{Z}$ do

centro de massa após o término da simulação

Figura 76 - Veículo robótico com o centro de massa representado

por uma linha contínua, após o término da simulação

Figura 77 - Veículo robótico retornando aos centros de massa

anteriores ao do término da simulação

Figura 78 - Veículo robótico avançando às posições dos

centros de massa até o término da simulação

Figura 79 - Exemplo de opção de terreno senos 
Figura 80 - Exemplo de opção de terreno flat

Figura 81 - Ilustração do terreno de grade regular retangular

Figura 82 - Exemplo da ordenação da malha do terreno

Figura 83 - Simulação utilizando a leitura de terreno do tipo mesh

(arquivo de malha "terrain_mesh2.msh")

Figura 84 - Exemplo de opção de terreno image (mapa de altura)

com o arquivo "heightmap3.bmp" definido para altura máxima "1.0”

Figura 85 - Exemplo de opção de terreno image (mapa de altura) com o arquivo "heightmap3.bmp" definido para altura máxima "5.0", com o veículo robótico superando o aclive do terreno em diferentes instantes

Figura 86 - Exemplo de opção de terreno image (mapa de altura) com o arquivo "heightmap52.bmp" definido para altura máxima "1.0"

Figura 87 - As três simulações utilizam o mesmo terreno criado a partir de um mapa de altura (arquivo "heightmap63.bmp"), sendo a ilustração

(a) sem nenhuma textura, com (b) textura "grass3.bmp" e

(c) com "tile31.bmp"

Figura 88 - Modelo de roda desenhado no software Blender com apresentação em vértices e arestas

Figura 89 - Modelos de roda com definição dos materiais

Figura 90 - Exemplos de chassi bem simples, ambos

com poucos detalhes e malha triangular

Figura 91 - Imagens da composição rodas mais chassi feita pelo simulador VirtualBotz 3D

Figura 92 - Visualização em Microsoft Excel do gráfico gerado pelo torque de uma das rodas do veículo robótico em uma simulação

Figura 93 - Visualização em Microsoft Excel do gráfico do sinal de controle PID de uma das rodas do veículo robótico durante uma simulação Figura 94 - Visualização em Microsoft Excel do gráfico do vetor $\mathbf{z}$ do centro de massa do veículo robótico em uma simulação Figura 95 - Visualização em Microsoft Excel do gráfico do vetor $\mathbf{Z}$ do centro de massa de uma das rodas dianteiras do veículo robótico, em vermelho, e uma das rodas traseiras, em azul, durante uma simulação 


\section{Lista de Tabelas}

Tabela 1 - Comparativo entre os simuladores avaliados 38

Tabela 2 - Biblioteca gráfica dos sistemas avaliados 42

Tabela 3 - Simuladores avaliados e suas bibliotecas de dinâmica 43

Tabela 4 - Coeficientes dos pneus para a “Fórmula Mágica” em [54],

valores em $k N \quad 68$

Tabela 5 - Coeficientes $a_{1}$ até $a_{8}$ para o pneu do veículo 69

Tabela 6 - Parâmetros do motor Magmotor [48] utilizado no robô VIVI 72

Tabela 7 - Parâmetros do veículo para solução analítica 81

Tabela 8 - Especificações do veículo robótico VIVI 88

Tabela 9 - Pastas do aplicativo 113

Tabela 10 - Localização e descrição de todas as bibliotecas do aplicativo 115

Tabela 11 - Exemplos de alguns dos dados dos fabricantes de motores [48] 165 


\section{Abreviaturas}

API

AS2TM

ASCII

ASL

AVI

CAD

CDTA

CUDA

CENPES

CLARAty

CODEC

CPU

DEM

FEM

GLUT

GNU GPL

GPGPU

GPU

GPS

IMU

MER

NASA

ODE

RCAST

RDS

RGS

ROAMS

RUR

TIN
Application Program Interface

AESCO Soft Soil Tire Model

American Standard Code for Information Interchange

Autonomous Systems Lab

Audio Video Interleaved

Computer Aided Design

Controle Dinâmico para Terrenos Acidentados

Compute Unified Device Architecture

Centro de Pesquisas e Desenvolvimento Leopoldo

Américo Miguez de Melo

Coupled-Layer Architecture for Robotic Autonomy

Contração do termo "COder-DECoder"

Central Processing Unit

Digital Elevation Model

Finite Element Modeller

The OpenGL Utility Toolkit

GNU General Public License

General-Purpose computing on Graphics Processing

Units

Graphics Processing Unit

Global Positioning System

Inertial Measurement Unit

Mars Exploration Rover

National Aeronautics and Space Administration

Open Dynamics Engine

Rover Chassis, Analysis and Simulation Tools

Microsoft Robotics Developer Studio

Rover Graphical Simulator

Rover Analisys, Modeling and Simulation

Rossum's Universal Robots

Triangular Irregular network 
UCP Unidade Central de Processamento

UM Universal Mechanisms

UML Unified Modelling Language

Win32

Plataforma de programação em janelas (Windows)

para 32 bits

WM2D Working Model 2D

.MSH Arquivo contendo coordenadas de uma coleção de polígonos

.MTL Arquivo de biblioteca de materiais referenciados pelos arquivos wavefront (.OBJ)

.OBJ Arquivo contendo coordenadas 3D de objeto em três dimensões 
“O valor das coisas não está no tempo que elas duram, mas na intensidade com que acontecem. Por isso existem momentos inesquecíveis, coisas inexplicáveis e pessoas incomparáveis.”

Fernando Sabino

“A vida não dá e nem empresta, não se comove e nem se apieda. Tudo quanto ela faz é retribuir e transferir aquilo que nós lhe oferecemos."

Albert Einstein

"Se as pessoas soubessem o quão duramente eu trabalhei para obter a minha habilidade, ela não pareceria tão maravilhosa depois de tudo." 